\title{
Femur Length and Head Circumference Ratio in Bangladeshi Fetuses
}

\author{
Sabrina Q. Rashid \\ SONOLAB, Center for Diagnostic Ultrasound, Dhaka, Bangladesh \\ Email: drsabrinaq@yahoo.com
}

Received 24 April 2014; revised 27 May 2014; accepted 5 June 2014

Copyright (C) 2014 by author and OALib.

This work is licensed under the Creative Commons Attribution International License (CC BY). http://creativecommons.org/licenses/by/4.0/

(c) (i) Open Access

\begin{abstract}
Objective: Fetal femur length and head circumference ratio is an important ratio for assessment of fetal skeletal growth. So far no study has been done on this ratio on Bangladeshi fetuses. Therefore this study was conducted. Method: This study was prospective and cross-sectional. A nomogram was prepared on well dated, singleton fetuses. A table and a graph were prepared by fitting Polynomial regression models. Results: Consecutive patients who fulfilled the required criteria were included. A total of 1223 subjects were studied. Percentiles, mean and 2 standard deviations were deduced by fitting models. The cubic model was a good fit to the data. Graphs of the data with superimposed curves were developed. Conclusion: For accurate assessment of fetal femur length/ head circumference ratio of Bangladeshi fetuses this chart can be used. Fetal skeletal growth abnormality and some skeletal anomalies can be detected by using this nomogram. This chart is more or less similar to the western chart.
\end{abstract}

\section{Keywords}

Fetal Femur Length/Head Circumference Ratio, Bangladesh

\section{Introduction}

To determine how the fetal parts are growing ratios are used. The ratio of fetal femur length with head circumference is an important ratio to assess growth of fetal skeleton and also it can be used to detect some skeletal anomalies. It is used to see if the fetal skeleton is growing proportionately. In Bangladesh no study of this ratio was done before this one.

Ratios can be a powerful method of comparing two parameters, as long as one of the parameters can be determined to be normal. The most useful ratios are those that remain constant through some period, thus making them easier to remember. Ratios of the cranium are constant throughout the second and third trimesters. Many 
ratios become relatively constant after mid-pregnancy [1].

Studies done on Bangladeshi population have found that fetal biometric measurements here are smaller than the Caucasian fetal measurements [2]-[7]. Therefore the western tables and graphs are not appropriate for use in Bangladesh. We need nomograms based on our own population studies. To this end this study was designed. In this study a table was prepared with the percentiles, mean and 2 standard deviations (2SD) of this ratio based on Bangladeshi population.

\section{Subjects and Method}

This was a prospective, cross sectional study. Each subject was therefore studied once only. The study was conducted on healthy gravid women, who were between 13 to 40 weeks of gestational age. Patients were Bengalis. Inclusion criteria were: patients with regular periods, no oral contraceptive taken for 3 months prior to conception, sure last menstrual period (LMP) with an ultrasonography scan done before 20 weeks of gestation to confirm the LMP date. No evidence of congenital anomalies. No maternal malnutrition or any medical, surgical or obstetric complication. No uterine anomaly or large fibroids. Well informed consent of the patients was obtained.

All patients included had ultrasound scans for measurements of the four main fetal parameters, which are, fetal biparietal diameter (BPD), head circumference (HC), abdominal circumference (AC) and femur length (FL) by standard methodology. The ultrasound machine employed was Aloka SSD1100 of Japan. A $3.5 \mathrm{MHz}$ curvilinear transducer was used for all examinations. All measurements were made by electronic calipers in mm.

The technique of measuring the femoral length involved an initial determination of the lie of the fetus and locating the femur. Once the femur was located, an attempt was made to define both ends of the calcified portion. The image was then frozen and with multidirectional electronic calipers, the calcified portion was measured. The aim was a femur which is finely outlined and had clear-cut ends [8]. After 32 menstrual weeks, the distal femoral epiphysis was visible but not included in the measurements [9].

The HC was obtained at a level that showed a smooth symmetric head, a well-defined midline echo, paired Thalami, the cavum septum pellucidum, and the third ventricle. For circumference measurements ellipse was used which opened to the outline of fetal head.

Statistical package for social science (SPSS) program in the computer software was used for data analysis. The ratio between the two fetal biometric parameters FL and HC were determined. A table and two graphs were produced after regression analysis and fitting models. Values were expressed as mean, 2 standard deviations and percentiles of the sample.

\section{Results}

A total of 1223, healthy gravid women, were studied. All of them satisfied the inclusion criteria. Mean maternal age was 26.95 ( \pm 4.49 ), the range was from 17 to 40 years age. The study population was predominantly middle class. $54.7 \%$ of the subjects were primipara and $45.3 \%$ were multipara. Mean maternal height was $156.73( \pm 5.1)$ $\mathrm{cm}$ and mean maternal weight taken in the first trimester was $57.92( \pm 9.2) \mathrm{kg}$. Mean BMI was $23.67( \pm 3.4)$.

Table 1 gives fitted percentiles of femur length/head circumference ratio. It gives the total number of cases in each week of gestation and estimated values of 3rd, 10th, 50th, 90th and 97th percentiles and the mean with 2 standard deviations of the data. At 13 weeks gestational age mean femur length/head circumference was 0.14 ( \pm $0.03)$, at 36 weeks it was $0.22( \pm 0.02)$ and at 40 weeks it was $0.23( \pm 0.02)$. Range of ratio was from 14 to 23 . The coefficient of multiple correlation $R^{2}=0.776$, $(p<0.001)$, and the cubic model gave a good fit to the data 2 standard deviations ranged from \pm 0.03 to \pm 0.02 .

Figure 1 shows the raw data for femur length/head circumference with fitted 3rd, 10th, 50th, 90th and 97th percentile curves superimposed on it. The values of the ratio increase as the pregnancy progresses. It gives $95 \%$ confidence interval (95\% CI).

Figure 2 shows assessment of goodness of fit of model for standard deviations of femur length/head circumference: Plot of standard deviation score against gestational age, showing expected 2 standard deviations.

\section{Discussion}

Ratios of two parameters are an important tool for comparison between the two parameters, but one parameter 
Table 1. Fitted percentiles of femur length/head circumference (FL/HC) ratio (Estimated values).

\begin{tabular}{|c|c|c|c|c|c|c|c|c|}
\hline Weeks of gestation (w) & No. of fetuses & 3rd & 10th & 50th & 90th & 97th & Mean & 2SD \\
\hline 13 & 15 & 0.11 & 0.12 & 0.14 & 0.16 & 0.17 & 0.14 & 0.03 \\
\hline 14 & 27 & 0.12 & 0.13 & 0.15 & 0.17 & 0.18 & 0.15 & 0.03 \\
\hline 15 & 32 & 0.13 & 0.14 & 0.16 & 0.18 & 0.19 & 0.16 & 0.03 \\
\hline 16 & 34 & 0.14 & 0.15 & 0.17 & 0.19 & 0.20 & 0.17 & 0.03 \\
\hline 17 & 27 & 0.15 & 0.16 & 0.18 & 0.20 & 0.21 & 0.18 & 0.03 \\
\hline 18 & 42 & 0.15 & 0.16 & 0.18 & 0.20 & 0.21 & 0.18 & 0.03 \\
\hline 19 & 34 & 0.16 & 0.17 & 0.19 & 0.21 & 0.22 & 0.19 & 0.03 \\
\hline 20 & 38 & 0.16 & 0.17 & 0.19 & 0.21 & 0.22 & 0.19 & 0.03 \\
\hline 21 & 43 & 0.18 & 0.19 & 0.20 & 0.21 & 0.22 & 0.20 & 0.02 \\
\hline 22 & 45 & 0.18 & 0.19 & 0.20 & 0.21 & 0.22 & 0.20 & 0.02 \\
\hline 23 & 39 & 0.19 & 0.20 & 0.21 & 0.22 & 0.23 & 0.21 & 0.02 \\
\hline 24 & 36 & 0.19 & 0.20 & 0.21 & 0.22 & 0.23 & 0.21 & 0.02 \\
\hline 25 & 42 & 0.19 & 0.20 & 0.21 & 0.22 & 0.23 & 0.21 & 0.02 \\
\hline 26 & 46 & 0.19 & 0.20 & 0.21 & 0.22 & 0.23 & 0.21 & 0.02 \\
\hline 27 & 44 & 0.20 & 0.21 & 0.22 & 0.23 & 0.24 & 0.22 & 0.02 \\
\hline 28 & 49 & 0.20 & 0.21 & 0.22 & 0.23 & 0.24 & 0.22 & 0.02 \\
\hline 29 & 50 & 0.20 & 0.21 & 0.22 & 0.23 & 0.24 & 0.22 & 0.02 \\
\hline 30 & 51 & 0.20 & 0.21 & 0.22 & 0.23 & 0.24 & 0.22 & 0.02 \\
\hline 31 & 52 & 0.20 & 0.21 & 0.22 & 0.23 & 0.24 & 0.22 & 0.02 \\
\hline 32 & 50 & 0.20 & 0.21 & 0.22 & 0.23 & 0.24 & 0.22 & 0.02 \\
\hline 33 & 55 & 0.20 & 0.21 & 0.22 & 0.23 & 0.24 & 0.22 & 0.02 \\
\hline 34 & 56 & 0.20 & 0.21 & 0.22 & 0.23 & 0.24 & 0.22 & 0.02 \\
\hline 35 & 59 & 0.20 & 0.21 & 0.22 & 0.23 & 0.24 & 0.22 & 0.02 \\
\hline 36 & 57 & 0.20 & 0.21 & 0.22 & 0.23 & 0.24 & 0.22 & 0.02 \\
\hline 37 & 54 & 0.21 & 0.22 & 0.23 & 0.24 & 0.25 & 0.23 & 0.02 \\
\hline 38 & 56 & 0.21 & 0.22 & 0.23 & 0.24 & 0.25 & 0.23 & 0.02 \\
\hline 39 & 55 & 0.21 & 0.22 & 0.23 & 0.24 & 0.25 & 0.23 & 0.02 \\
\hline 40 & 35 & 0.21 & 0.22 & 0.23 & 0.24 & 0.25 & 0.23 & 0.02 \\
\hline
\end{tabular}

has to be normal for an accurate comparison. Statistical methods of analysis have now improved, so good quality fetal nomograms can be developed.

In the present study, a model was fitted to the data and estimated values were presented. At 13 weeks gestational age mean FL/HC was $0.14( \pm 0.03)$ and at 40 weeks it was $0.23( \pm 0.02)$, with $95 \%$ CI of 0.21 to 0.25 . $R^{2}$ $=0.776$, $(p<0.001)$. Plot of standard deviation scores against gestational age, showed expected 2SD. The 3rd, 10th, 50th, 90th and 97th percentile curves derived by fitting a model were superimposed on the scatter chart of the study population and the cubic model gave a good fit to the data. It covered $95 \%$ of the population.

The ratio in this study was low in the beginning and gradually increased till term. At 13 weeks it was 14 ( \pm 3 ) and at 40 weeks it was $23( \pm 2)$. In a Western study at 15 weeks the ratio was $16( \pm 2)$ and at 40 weeks, it was 21 $( \pm 2)[10]$. So it is more or less similar to the present study. 


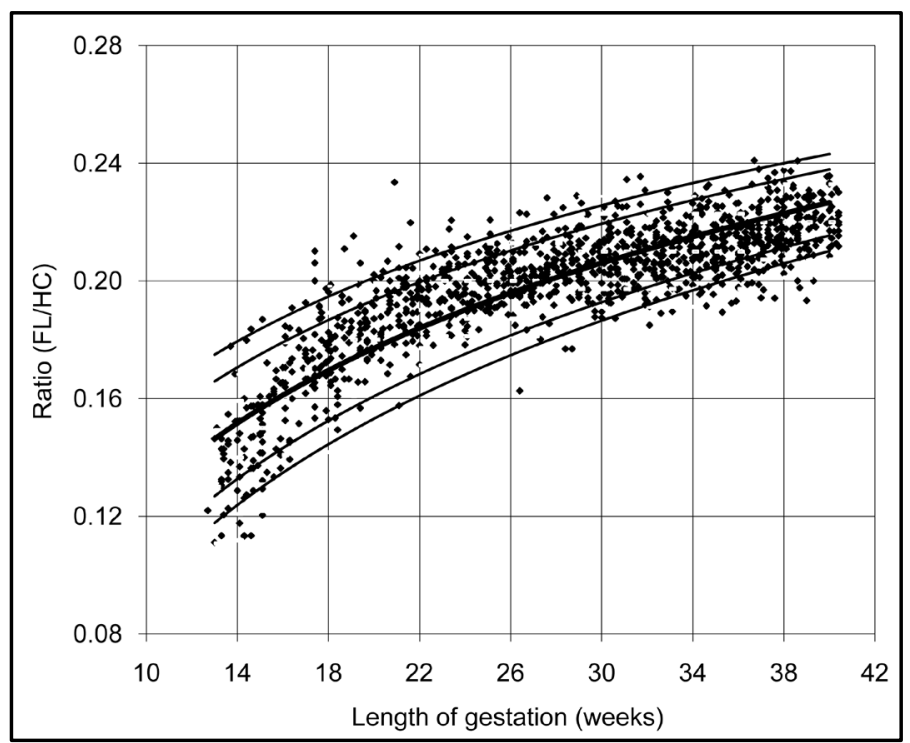

Figure 1. Raw data for femur length/head circumference (FL/HC) ratio with fitted $3^{\text {rd }}, 10^{\text {th }}, 50^{\text {th }}, 90^{\text {th }}$ and $97^{\text {th }}$ percentiles.

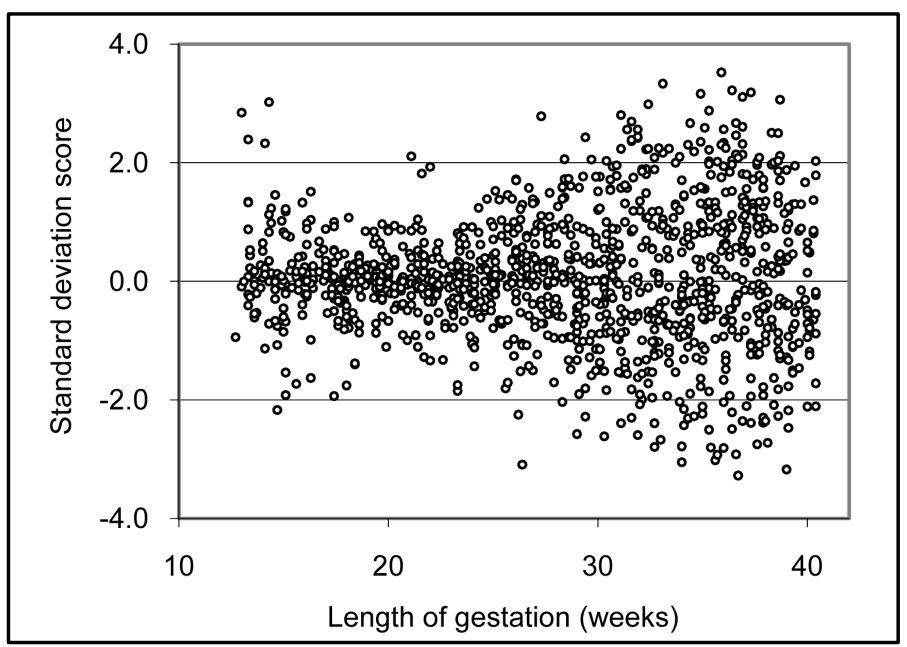

Figure 2. Assessment of fit of model for FL/HC plotted: Plot of standard deviation score against gestational age, showing expected 2SD.

\section{Limitations}

The study population was predominantly middle income group as poor women mostly deliver at home because of socio-economic reasons. Even when some go to the doctors they are unable to tell their LMP precisely because of illiteracy, but this was mandatory for this study.

\section{Conclusion}

In this study fetal FL/HC ratio has been prepared for the first time in Bangladesh. The value of this ratio is somewhat similar to the western ratio. This is unlike the individual fetal biometric parameters which differ from the western data near term.

\section{References}

[1] DuBose, T.J. (1996) Size/Age Analysis. In: DuBose, T.J., Ed., Fetal Sonography, 1st Edition, WB Saunders, Philadel- 
phia, 95-156.

[2] Moslem, F., Latifa, S., Iffatara, B., Shamsuddin, A.K., Nasreen, M., Momen, A., et al. (1996) Relation of BPD with Gestational Age in Bangladeshi Fetus. Bangladesh Journal of Ultrasography, 3, 3-8.

[3] Bala, K.G. (1991) Ultrasound Assessment of Fetal BPD during Normal Pregnancy in Bangladeshi Women and Review of Literatures. Bangladesh Journal of Ultrasography, 1, 3.

[4] Rashid, S.Q. (1999) A Study Correlating the Menstrual Age and Fetal Age by Ultrasonography in Bangladeshi Population. Bangladesh Journal of Ultrasography, 6, 3-8.

[5] Rashid, S.Q. (2006) Gestational Age Estimation from Fetal Head Circumference in Bangladesh. Ultrasound in Medicine and Biology, 32, 163-164.

[6] Rashid, S.Q. and Khatun, S. (2001) A Study of Estimated Fetal Weights by Ultrasound in Bangladesh and Its Correlation with Birth Weights. Journal of Bangladesh College of Physicians and Surgeons, 19, 47-51.

[7] Rashid, S.Q. (2010) Gestational Age Prediction by Femur Length in Bangladeshi Population. Journal of Bangladesh College of Physicians and Surgeons, 28, 163-166.

[8] O’Brien, G.D., Queenan, J.T. and Campbell, S. (1981) Assessment of Gestational Age in the Second Trimester by Real-Time Ultrasound Measurement of the Femur Length. American Journal of Obstetrics \& Gynecology, 139, 540545.

[9] Queenan, J.T., O’Brien, G.D. and Campbell, S. (1980) Ultrasound measurement of fetal limb bones. American Journal of Obstetrics \& Gynecology, 138, 297-302.

[10] Hadlock, F.P., Harrist, R.B., Shah, Y. and Park, S.K. (1984) The Femur Length/Head Circumference Relation in Obstetric Sonography. Journal of Ultrasound in Medicine, 3, 439-442. 